\title{
Solving of Linear Time Invariance Quadratic Optimal Control Systems using Chebyshev Scaling Function
}

\author{
Adane Akate \\ College of natural and computational sciences, Department of mathematics, Mekdela Amba \\ University, Dessie, Ethiopia
}

\begin{abstract}
In this paper we have studied the linear time invariance optimal control problems with quadratic performance index, and approximated control variable, state variable and performance index using Chebyshev scaling function method with unknown coefficients. The linear time invariant problems were parameterized based on control-state parameterization technique such that the objective function and the constraints are casted in terms of state variable and control variable. This method was converting the linear time invariance quadratic optimal control problems into quadratic programming problems and the converted problems were solved using MATLAB. Hence we increase the order of polynomial (M), and then the computational results of the proposed methods gave better results.
\end{abstract}

Keywords: Optimal control, Chebyshev scaling function, operational matrix integration.

DOI: $10.7176 / \mathrm{MTM} / 9-7-01$

Publication date: July $31^{\text {st }} 2019$

\section{Introduction}

Optimal control is a science that deals with linear and nonlinear optimal control problems (OCPs) and the main objective of optimal control is to determine control function that will the cause of a systems (plant) to satisfy the physical constraints and at the same time extremize (maximize or minimize) a performance criterion (performance index or cost function) [1]. According to [2] the value of state variable decreases when the time interval increases. According to [3] the value of control variable increases when the time interval increases in the case of linear time invariant optimal control problems. Analytical solutions of optimal control problems are not always available, thus finding an approximate solution is at least the most logical way to solve them.

Linear optimal control is a special sort of optimal control where the system that is controlled was assumed linear and the controller, the device that generates the optimal control is also constraints to be linear [4].In the work of [5] Chebyshev-based representation of the state variable has been proposed for designing optimal control variables of unconstrained, linear, dynamic systems with quadratic performance indices. [6] discussed the Chebyshev wavelet to solve the linear quadratic optimal control problem with terminal constraints. The method is based on converting the OCPs into mathematical programming and he used the operational matrix of differentiation. [7] Proposed a method to solve the linear-quadratic and the nonlinear OCPs by using Chebyshev polynomials to parameterize some of the state variables, and then the remaining state variables and the control variables are determined from the state equations.

In this paper we propose a system made up of the special class of optimal control problem is linear time invariant optimal control problem, with quadratic objective functions and the constraints is linear. To find numerical solutions of linear time quadratic optimal control problem , how Chebyshev scaling function with polynomial of degree three can be adapted 
and applied to these optimization problems but these two methods defined on the time interval $\mathrm{t} \in[0,1]$.

\section{Methods}

Using Chebyshev scaling function convert the linear time invariant quadratic optimal control problems into quadratic programming problems. Then the converted quadratic programming problems were solved using MATLAB and we calculate the state variable, control variable and performance index.

\section{Chebyshev Scaling Function}

We will introduce the definition, basis of Chebyshev scaling function. This basis will be the basic of this project in the following section. Chebyshev scaling function is used to for approximate both state variables and control variables because the optimal solution of state variables and control variables are a function of time so it is better to use this function for quadratic optimal control problems and Chebyshev scaling function can be defined is as follows

$$
\begin{aligned}
& \Psi_{\mathrm{nm}}(\mathrm{t})=\left\{\begin{array}{cl}
\frac{\alpha_{\mathrm{m}} 2^{(\mathrm{k}-1) / 2}}{\sqrt{\pi}} \mathrm{T}_{\mathrm{m}}\left(2^{\mathrm{k}} \mathrm{t}-2 \mathrm{n}+1\right), \frac{\mathrm{n}-1}{2^{\mathrm{k}-1}} \leq \mathrm{t} \leq \frac{\mathrm{n}}{2^{\mathrm{k}-1}} \\
0, \quad \text { otherwise }
\end{array}\right. \\
& \text { where } \alpha_{\mathrm{m}}= \begin{cases}\sqrt{2} & , \mathrm{~m}=0 \\
2 & , \mathrm{~m}=1,2,3, \ldots\end{cases}
\end{aligned}
$$

Here, $\mathrm{T}_{\mathrm{m}}(\mathrm{t})$ are the Chebyshev polynomials of order $\mathrm{m}$,

$$
\begin{gathered}
\mathrm{T}_{0}(\mathrm{t})=1, \\
\mathrm{~T}_{1}(\mathrm{t})=\mathrm{t} \\
\mathrm{T}_{2}(\mathrm{t})=2 \mathrm{t}^{2}-1 \\
\mathrm{~T}_{3}(\mathrm{t})=4 \mathrm{t}^{3}-3 \mathrm{t} \\
\mathrm{T}_{\mathrm{m}+1}(\mathrm{t})=2 \mathrm{tT}_{\mathrm{m}}(\mathrm{t})-\mathrm{T}_{\mathrm{m}-1}(\mathrm{t}), \mathrm{m}=1,2,3, \ldots
\end{gathered}
$$

\subsection{Operational Matrix of Integration (OMI) for Chebyshev Scaling Function}

Let $\mathrm{P}$ be an operational matrix of integration which is obtained from the integral of Chebyshev scaling function from 0 to $t$ and these matrices play an important role to modeling the problems. According to [8], this matrix is used to change the system of state equations into a set of algebraic equations which can be solved using software. This is represented as follows:

$$
\begin{gathered}
\int_{0}^{\mathrm{t}} \Psi(\tau) \mathrm{d} \tau=P \Psi(\mathrm{t}) \\
\Psi(\mathrm{t})=\left[\Psi_{1 \mathrm{~m}}(\mathrm{t}), \Psi_{2 \mathrm{~m}}(\mathrm{t}), \Psi_{3 \mathrm{~m}}(\mathrm{t}), \ldots, \Psi_{2^{\mathrm{k}-1} \mathrm{~m}}(\mathrm{t})\right]^{\mathrm{T}}
\end{gathered}
$$

So the matrix $\mathrm{P}$ is the cofficent of $\Psi(\mathrm{t})$

$\mathrm{P}=\left[\begin{array}{ll}\mathrm{C} & \mathrm{S} \\ \mathrm{O} & \mathrm{C}\end{array}\right]$

where $\mathrm{P}$ is a $\left(2^{\mathrm{K}-1}(\mathrm{M}+1) \times 2^{\mathrm{K}-1}(\mathrm{M}+1)\right)$ operational matrix of integration and $\mathrm{O}, \mathrm{C}, \mathrm{S}$ are $(\mathrm{M}+1) \times(\mathrm{M}+1)$ matrices and give 


$$
\begin{aligned}
& \mathrm{S}=\frac{\sqrt{2}}{2^{\mathrm{K}-1}}\left[\begin{array}{ccccc}
\frac{1}{\sqrt{2}} & 0 & 0 & \cdots & 0 \\
0 & 0 & 0 & \cdots & 0 \\
\frac{-1}{3} & 0 & 0 & \cdots & 0 \\
0 & 0 & 0 & \cdots & 0 \\
\frac{-1}{15} & 0 & 0 & \cdots & 0 \\
\vdots & \vdots & \vdots & \ddots & 0 \\
\frac{-1}{\mathrm{M}(\mathrm{M}-2)} & 0 & 0 & \cdots & 0
\end{array}\right] \\
& \mathrm{C}=\frac{1}{2^{\mathrm{k}-1}}\left[\begin{array}{cccccccc}
\frac{1}{2} & \frac{1}{2 \sqrt{2}} & 0 & 0 & \cdots & 0 & 0 & 0 \\
\frac{-1}{4 \sqrt{2}} & 0 & \frac{1}{8} & 0 & \cdots & 0 & 0 & 0 \\
\frac{-1}{3 \sqrt{2}} & \frac{-1}{4} & 0 & \frac{1}{12} & \cdots & 0 & 0 & 0 \\
\vdots & \vdots & \vdots & \vdots & \ddots & \ddots & \ddots & 0 \\
\frac{-1}{2 \sqrt{2}(\mathrm{M}-1)(\mathrm{M}-3)} & 0 & 0 & 0 & \cdots & \frac{-1}{4(\mathrm{M}-3)} & 0 & \frac{-1}{4(\mathrm{M}-1)} \\
\frac{-1}{2 \sqrt{2} \mathrm{M}(\mathrm{M}-2)} & 0 & 0 & 0 & \cdots & 0 & \frac{-1}{4(\mathrm{M}-2)} & 0
\end{array}\right]
\end{aligned}
$$

$\mathrm{O}$ is matrix that all entire elements are zeros

\subsection{Continuity Test of Chebyshev Scaling Function}

To insure the continuity of the state variables between the different sections so we must add constraints. There are $2^{\mathrm{k}-1}-1$ points at which the continuity of the state variables has to ensure. These points are

$$
\mathrm{t}_{\mathrm{i}}=\frac{\mathrm{i}}{2^{\mathrm{k}-1}} \quad, \mathrm{i}=1,2, \ldots, 2^{\mathrm{K}-1}-1
$$

In addition, there is $\left(2^{\mathrm{K}-1}-1\right) \mathrm{s}$ equality constraints can be given as follows

$$
\left(\mathrm{I}_{\mathrm{s}} \otimes \Phi^{\prime}\right) \mathrm{a}=\mathrm{o}_{\left(2^{\mathrm{K}-1}-1\right)_{\mathrm{s} \times 1}}
$$

However, the dimension of continuity matrix $\Phi^{\prime}$ or ensured constraints is $\left(2^{\mathrm{K}-1}-1\right) \times$ $2^{\mathrm{K}-1}(\mathrm{M}+1)$.

\subsection{Quadratic Programming Problem for Chebyshev Scaling Function}

In this section, we try to construct the quadratic form of optimal control problem can be easily solved using MATLAB code.

From the integration of state equation can be rewritten using operational matrix of integration and unknown coefficients in the following form.

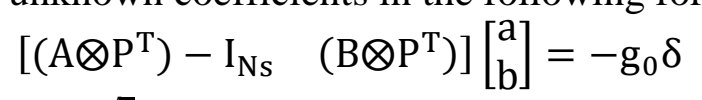

where $\delta=\frac{\sqrt{\pi}}{2}$, A is an $\mathrm{n} \times \mathrm{n}$ matrix and $\mathrm{B}$ is an $\mathrm{n} \times \mathrm{m}$ are the coefficient matrices of state variable and control variable from the state equation respectively, $\mathrm{g}_{0}$ is the initial column vector, $P$ is operational matrix of integration with the dimension of $2^{\mathrm{K}-1}(\mathrm{M}+1), \mathrm{I}_{\mathrm{Ns}}$ is identity matrix with the dimension of $2^{\mathrm{K}-1}(\mathrm{M}+1)$ and $\mathrm{a}, \mathrm{b}$ are unknown coefficients.

By combining equations (5) and (6), we get the following form of equality constraints

$$
\left[\begin{array}{cc}
\left(\mathrm{A} \otimes \mathrm{P}^{\mathrm{T}}\right)-\mathrm{I}_{\mathrm{Ns}} & \left(\mathrm{B} \otimes \mathrm{P}^{\mathrm{T}}\right) \\
\mathrm{I}_{\mathrm{S}} \otimes \Phi^{\prime} & \mathrm{o}_{\left(2^{\mathrm{K}-1}-1\right)_{\mathrm{s} \times \mathrm{Nr}}}
\end{array}\right]\left[\begin{array}{l}
\mathrm{a} \\
\mathrm{b}
\end{array}\right]=\left[\begin{array}{c}
-\mathrm{g}_{0} \delta \\
\mathrm{o}_{\left(2^{\mathrm{K}-1}-1\right)_{\mathrm{s} \times 1}}
\end{array}\right]
$$

Then compact quadratic form is as follows:

$$
\begin{array}{ccc}
\min _{\mathrm{z}} \mathrm{z}^{\mathrm{T}} \mathrm{Hz} & \\
\text { Subject to } & \mathrm{Fz}=\mathrm{h} & \\
& & \text { where, } \quad \mathrm{z}=\left[\begin{array}{l}
\mathrm{a} \\
\mathrm{b}
\end{array}\right]
\end{array}
$$




$$
\begin{gathered}
H=\left[\begin{array}{cc}
R R \otimes Q & O_{N s \times N r} \\
O_{N r \times N s} & R R \otimes R
\end{array}\right] \\
F=\left[\begin{array}{cc}
\left(A \otimes P^{T}\right)-I_{N s} & \left(B \otimes P^{T}\right) \\
\left(I_{s} \otimes \Phi^{\prime}\right) & \mathrm{O}_{\left(2^{K-1}-1\right)_{s \times N r}}
\end{array}\right] \\
h=\left[\begin{array}{c}
-\mathrm{g}_{0} \delta \\
\mathrm{O}_{\left(2^{\mathrm{K}-1}-1\right)_{s \times 1}}
\end{array}\right]
\end{gathered}
$$

\section{Linear Time Invariance Quadratic Optimal Control Problem}

Before approximating the states variable and control variable, it is necessary to transform the time in the optimal control problem $t \in\left[\mathrm{t}_{0}, \mathrm{t}_{\mathrm{f}}\right]$ into the interval $\tau \in[0,1]$; because Chebyshev scaling function [3] are defined on the interval $\tau \in[0,1]$.

Then we can obtain by using

Then the transformed optimal control problem is

$$
\tau=\frac{t}{t_{f}} \quad \text { so } \quad d t=t_{f} d \tau
$$

$$
\begin{aligned}
& \min J=t_{f} \int_{0}^{1}\left(x^{T}(t) Q x(t)+u^{T}(t) \operatorname{Ru}(t)\right) d \tau \\
& \text { subject to } \frac{d x(t)}{d \tau}=t_{f}(\operatorname{Ax}(t)+B u(t))
\end{aligned}
$$

\subsection{Approximation of State and Control Variable Using Chebyshev Scaling Function}

The basic idea of Chebyshev scaling function is to approximate the state and control variables for the linear time invariance quadratic optimal control problems by using a finite series is as follows.

$\begin{array}{ll}\mathrm{x}_{\mathrm{i}}(\mathrm{t})=\sum_{\mathrm{n}=1}^{2^{\mathrm{k}-1}} \sum_{\mathrm{m}=0}^{\mathrm{M}} \mathrm{a}^{\mathrm{i}}{ }_{\mathrm{nm}} \Psi_{\mathrm{nm}}(\mathrm{t}), & \mathrm{i}=1,2,3, \ldots, \mathrm{s} \\ \mathrm{u}_{\mathrm{i}}(\mathrm{t})=\sum_{\mathrm{n}=1}^{2^{\mathrm{k}-1}} \sum_{\mathrm{m}=0}^{\mathrm{M}} \mathrm{b}^{\mathrm{i}}{ }_{\mathrm{nm}} \Psi_{\mathrm{nm}}(\mathrm{t}), & \mathrm{i}=1,2,3, \ldots, \mathrm{r}\end{array}$

\subsection{Approximation of performance index via Chebyshev Scaling Function}

We can approximate the performance index of linear time invariance quadratic optimal control problems using Chebyshev scaling function, substitute equations (10) and (11) into the objective function. We get

$$
J=\frac{1}{2} \int_{0}^{1} a^{T}\left(\Psi(t) \otimes I_{s}\right) Q\left(I_{s} \otimes \Psi^{T}(t)\right) a+b^{T}\left(\Psi(t) \otimes I_{r}\right) R\left(I_{r} \otimes \Psi^{T}(t)\right) b d t
$$

It can be simplify as

$$
J=\frac{1}{2} \int_{0}^{1} a^{T}\left(\Psi(t)\left(\Psi^{T}(t) \otimes Q\right) a+b^{T}\left(\Psi(t)\left(\Psi^{T}(t) \otimes R\right) b d t\right.\right.
$$

By using equation (12), we get

It can be write in a quadratic form

$$
\text { then } \quad J=\frac{1}{2}\left(a^{T}(R R \otimes Q) a+b^{T}(R R \otimes R) b\right)
$$




$$
J=\left[\begin{array}{ll}
a^{T} & b^{T}
\end{array}\right]\left[\begin{array}{ll}
R R \otimes Q & O_{N s \times N r} \\
O_{N r \times N s} & R R \otimes R
\end{array}\right]\left[\begin{array}{l}
a \\
b
\end{array}\right]
$$

Then the general steps to approximate state, control variable and performance indices using Chebyshev scaling function with unknown coefficients of $a$ and $b$.

Step (1): Define the unknown coefficients of state and control variables according to $\mathrm{K}$ and M. The unknown coefficients will be as follows.

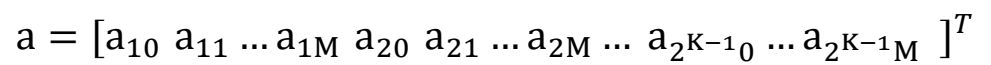

$$
\begin{aligned}
& b=\left[\begin{array}{llllllllllll}
b_{10} & b_{11} & \ldots & b_{1 M} & b_{20} & b_{21} & \ldots & b_{2 M} & \ldots & b_{2^{K-1}} & \ldots & b_{2^{K-1} M}
\end{array}\right]^{T}
\end{aligned}
$$

Step (2): Generate the Chebyshev scaling function depending on $\mathrm{K}$ and $\mathrm{M}$.

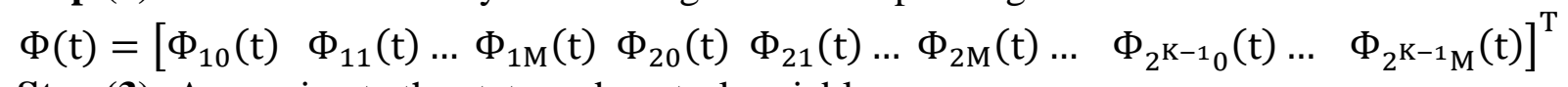

Step (3): Approximate the state and control variables.

Step (4): Find the vector of initial condition

$$
\begin{aligned}
& \mathrm{x}(\mathrm{t})=\sum_{\mathrm{n}=1}^{2^{\mathrm{K}-1}} \sum_{\mathrm{m}=0}^{\mathrm{M}} \mathrm{a}_{\mathrm{nm}} \Phi_{\mathrm{nm}} \\
& \mathrm{u}(\mathrm{t})=\sum_{\mathrm{n}=1}^{2^{\mathrm{K}-1}} \sum_{\mathrm{m}=0}^{\mathrm{M}} \mathrm{b}_{\mathrm{nm}} \Phi_{\mathrm{nm}}
\end{aligned}
$$

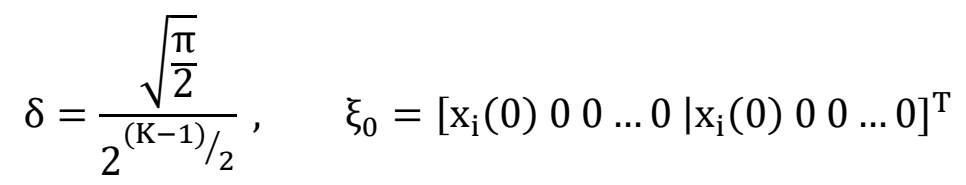

Step (5): Find the points of ensured continuity and the continuity matrix.

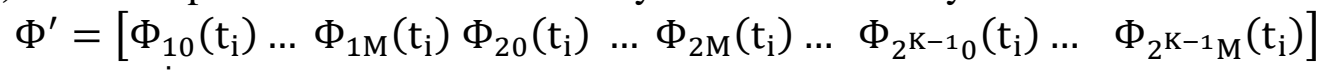

$$
\begin{aligned}
& \text { where } t_{i}=\frac{i}{2^{\mathrm{K}-1}}, i=1,2,3, \ldots, 2^{\mathrm{K}-1}-1
\end{aligned}
$$

Step (6): Determine the quadratic programming problems or equation (8)

Step (7): Solve for the equation (8) or quadratic programming problem using MATLAB.

\section{Example}

Find the optimal control $\mathrm{u}^{*}(\mathrm{t})$ and state variable $\mathrm{x}^{*}(\mathrm{t})$ which minimize the performance index.

$$
\begin{aligned}
& \min J=\frac{1}{2} \int_{0}^{1}\left(x^{2}(t)+u^{2}(t)\right) d t \\
& \text { Subject to } x^{\prime}(t)=-x(t)+u(t), \quad x(0)=1
\end{aligned}
$$

Fig. (1), shows that as the approximation value of state variable and control variable in the linear time invariant quadratic optimal control system, were presented using Chebyshev scaling function. We have considered parameter values $K=2$, with polynomial (M) of degree three. We observed that as the time interval, $t \in(0,1)$ the value of state variable decreases and control variable increases. 


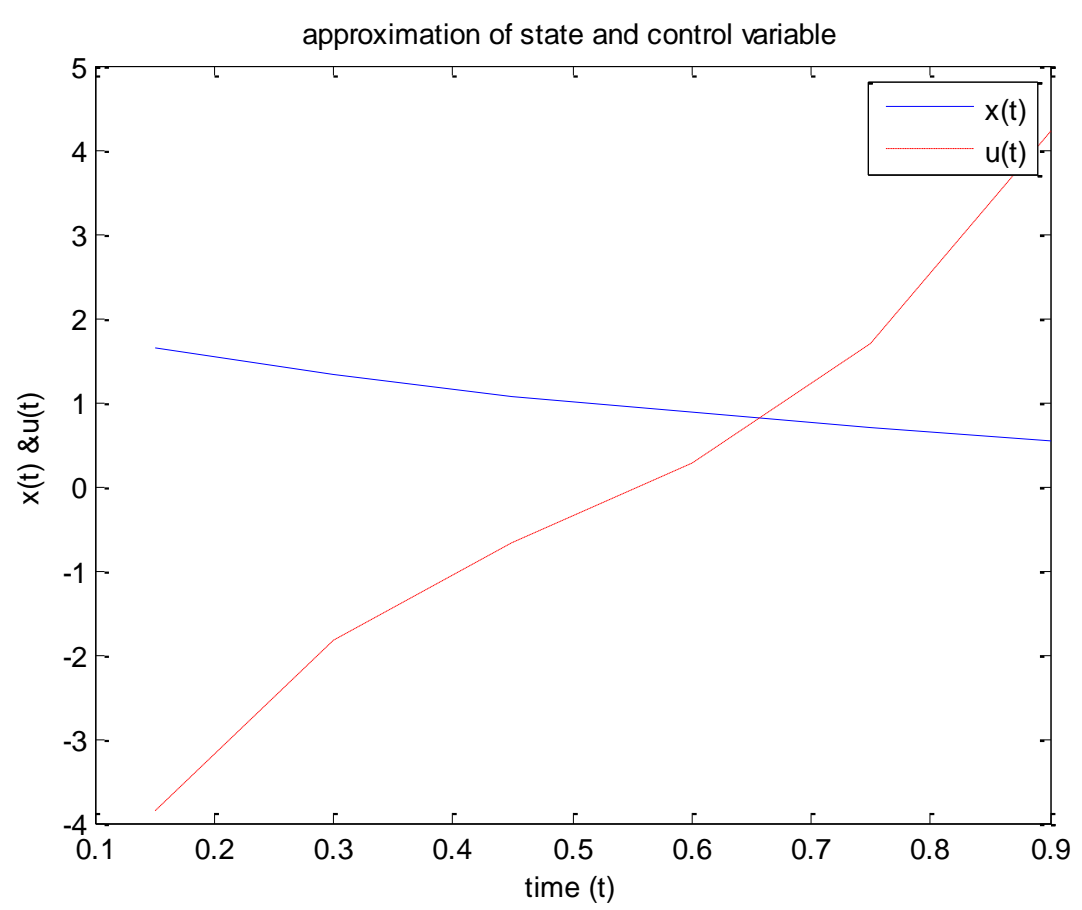

Figure 1: The approximation of state and control variable using Chebyshev scaling function In this paper we have study the linear time invariance optimal control problems with quadratic performance index, and approximated control variable, state variable and performance index using Chebyshev scaling function.

Table 1. Approximations of performance index using Chebyshev scaling function of example.

\begin{tabular}{|c|c|c|c|}
\hline \multicolumn{3}{|c|}{ Optimal value (Zeid and Yousefi, 2016) } & 0.1929092981 \\
\hline \multirow[t]{2}{*}{ Order of poly } & \multicolumn{2}{|c|}{ Performance index $(\mathrm{J})$} & \\
\hline & Chebyshev & Error & \\
\hline $\mathrm{M}=1$ & 0.196333244960920 & $3.42 \times 10^{-3}$ & \\
\hline $\mathrm{M}=2$ & 0.193000989090520 & $9.17 \times 10^{-5}$ & \\
\hline$M=3$ & 0.192934130143056 & $2.48 \times 10^{-5}$ & \\
\hline
\end{tabular}

Table (1) which shows the approximations of performance index using Chebyshev scaling function.

Table (1) shows that, for the polynomial of scaling function with degree three and the time interval $t \in[0,1]$, the absolute error is less (almost zero) with the Chebyshev scaling functions method, which yields a small error compared with the optimal solutions of the problem.

\section{Conclusion}

In this work, we have considered the linear time invariance quadratic optimal control problems. The results obtained for the linear time optimal control systems with quadratic performance index using Chebyshev scaling functions works for finding the approximation 
values of the state variable $\mathrm{x}(\mathrm{t})$, the control variable $\mathrm{u}(\mathrm{t})$ and performance indices. It was also deduced that the polynomial of scaling function with degree three and the time interval $t \in$ $[0,1]$, the absolute error is less (almost zero) with the Chebyshev scaling functions method. The result shows that the linear time invariance quadratic optimal control problems are solved by Chebyshev scaling functions.

\section{References}

[1] M. J. Wadi, Solving quadratic optimal control problems using Legendre scaling function and iterative technique, 2011.

[2] M. Behroozifar, S.A.Yousefi , and N. Ranjbar, Numerical solution of optimal control of time-varying singular systems via operational matrices. International Journal of Engineering transactions A: Basic, 2014, 27(4): 523-532.

[3] M. R., Fatehi, M. A. Vali, and M. Samavat, State analysis and optimal control of linear time-invariant scale systems using the Legendre wavelets. Canadian Journal on Automation, Control \& Intelligent Systems, 2012, 3(1): 1-7.

[4] P. G. Hans, Optimal control with engineering applications, $1^{\text {st }}$ edition. Zurich, switzerland, Spinger-Verlag Inc., Berlin, Heidelberg, 2007.

[5] M. L. Nagurka, S. K. Wang, and V. Yen, Linear quadratic optimal control system design by Chebyshev-based state parameterization. Department of Mechanical Engineering Carnegie Mellon University Pittsburgh, Pennsylvania, 1991, USA.

[6] H. Jaddu, Optimal control of time-varying linear systems using wavelets. Japan Advanced Institute of Science and Technology, 2006, 923-1292.

[7] H. Jaddu, and E. Shimemura, Computation of optimal control trajectories using Chebyshev polynomials: parameterization, and quadratic programming. Optimal Control Applications and Methods, 1999, 20(1): 21-42.

[8] M. Ghasemi, and K. M. Tavassoli, Numerical solution of time-varying delay systems by Chebyshev wavelets. Appl. Math. Model, 2011, 35(11): 5235-5244. 